\title{
BERNARD RIBÉMONT
}

\author{
Université d'Orléans
}

\section{UN « ROMAN IDYLLIQUE » DU XV ${ }^{\mathrm{e}}$ SIÈCLE ET LE DROIT MATRIMONIAL : PARIS ET VIENNE DE PIERRE DE LA CÉPÈDE}

\begin{abstract}
Bernard Ribémont, $U n$ "roman idyllique » $d u X V^{e}$ siècle et le droit matrimonial : "Paris et Vienne » de Pierre de La Cépède [The fifteenth century "idyllic romance" and matrimonial law - Paris et Vienne of Pierre de La Cépède], Studia Romanica Posnaniensia, Adam Mickiewicz University Press, Poznań, vol. XXXVIII/1: 2011, pp. 3-12. ISBN 978-83-232-2279-8. ISSN 0137-2475. DOI 10.2478/ v10123-011-0001-2.
\end{abstract}

Since the pioneering work of Myrrha Lot-Borodine identifying a particular form of the novel, with Floire et Blancheflor as a founding text where a much more central role is given to women. I would like here to analyze Paris et Vienne a text that critics had little studied, from the perspective of family law and matrimonial issues such that can be analyzed through the fiction that proposes to 1432, Pierre La Cépède. I will work on the short version, because condensed, only shows that the more deep springs of the text.

Depuis les travaux pionniers de Myrrha Lot-Borodine ${ }^{1}$, la critique a identifié un genre romanesque particulier', dont le texte 'fondateur' serait Floire et Blancheflor, genre qui fait porter l'accent bien plus sur des questions amoureuses que sur des prouesses chevaleresques, et marquerait ainsi un certain déclin des valeurs guerrières au profit d'un rôle beaucoup plus central donnée aux femmes. Je voudrais ici, en considérant un texte que la critique n'a que peu étudié3, considérer la relation entre le héros, Paris, et la fille du dauphin de Viennois, Vienne, du point de vue du droit familial et de la question matrimoniale tels que l'on peut les analyser à travers la fiction que propose, vers 1432, un auteur d'origine marseillaise, Pierre de La Cépède ${ }^{4}$.

${ }^{1}$ M. Lot-Borodine, Le Roman idyllique au Moyen Âge, Paris, Picard, 1913 [Genève, 1972].

${ }^{2}$ Voir le thème principal du numéro 20 des Cahiers de Recherches Médiévales et Humanistes (dir. M. Szkilnik) (déc. 2010). Cf. aussi L. Pierdominici, La Bouche et le corps. Images littéraires du quinzième siècle français, Paris, Champion, 2003.

${ }^{3}$ Voir la bibliographie dans l'édition du texte : Pierre de La Cépède, Paris et Vienne (version courte), éd. A.M. Babbi, Università di Verona, Milano, Francoangeli, 1992, pp. 149-152. J.J. Vincensini, « Désordre de l'abjection et ordre de la courtoisie : le corps abject dans Paris et Vienne de Pierre de La Cépède », 1992 Medium Aevum, 68, 1999, pp. 292-304.

${ }^{4}$ Cette œuvre connut un véritable succès et fut traduite en plusieurs langues, du moins la version courte. Pierre, dans le prologue, indique que lui-même a traduit une version provençale issue d'une version catalane. Antoine de La Sale, qui aurait peut-être rencontré Pierre, s'intéresse aussi à cette histoire (cf. S. Lefèvre, Antoine de la Sale. La fabrique de l'œuvre et de l'écrivain. Suivi de l'édition critique $d u$ Traité des anciens et des nouveaux tournois, Genève, Droz, 2006. pp. 155-156). 
Je travaillerai sur la version courte qui, parce que condensée, n'en révèle que mieux les ressorts profonds du texte.

Le début du roman se veut contextualisant, dans la mesure où l'auteur indique d'entrée de jeu que l'action qu'il va narrer se déroule « au temps du roy Charles roy de France, courant l'an de l'incarnacion de nostre seigneir Jhesu Crist Mil CCLXXJ, en la terre du Daulphiné en une cité qui s'appelle Vienne »; en cette terre règne un certain Godefroy d'Alençon, du lignage du roi de France précise le narrateur. Ce 'contexte' historique est évidemment faux ; en 1271, c'est Philippe III qui règne en France et ce dauphin Godefroy d'Alençon n'a jamais existé. Cependant, cette façon de présenter l'œuvre sur un effet de realia marque celle-ci d'une dimension de sérieux et de didactisme ; cette introduction en effet entre en écho, non sans humour peut-être, avec ne nombreux textes de la fin du Moyen Âge qui sont effectivement précisément situés, à tel point que la datation, la précision géographique, réelles ou fantaisistes, s'érigent en topos.

Godefroy, selon le narrateur, est un homme de grande sagesse, très écouté du roi de France, au lignage duquel il appartient lui-même. Connotation intéressante, au XV $\mathrm{X}^{\mathrm{e}}$ siècle, à une époque où, et ce depuis 1349 , le titre de dauphin est réservé à l'héritier du royaume. On pourrait voir là une revendication 'régionaliste' de l'auteur. En tout état de cause, cette importance donnée au Dauphiné s'inscrit, il me semble, dans la mise en place de la diégèse ; en effet, la fille de Godefroy et de sa femme sera nommée Vienne, du nom de la capitale du Dauphiné. Ce choix onomastique me paraît signifiant. En effet, le roman s'ouvre, selon l'expression de Jean-Jacques Vincensini, sur un « trouble généalogique $»^{5}$ : le dauphin et sa femme n'arrivent pas à avoir d'enfant, donc pas d'héritier. Mais, à force de prières, Dieu, au bout de sept ans, exauce ce couple de bons chrétiens et une fille vient au monde. Celle-ci est donc la seule héritière de Godefroy. Son nom prend donc une signification particulièrement forte : l'onomastique associe en effet, de façon très étroite, dans une véritable osmose, la fille et la terre. On peut voir dans ce choix de l'auteur deux raisons : la première tient à une tradition et l'on sait que les textes de la fin du Moyen Âge se plaisent aux 'réexploitations décalées', en particulier de thèmes et motifs du roman chevaleresque ou de la chanson de geste. Or l'association de la femme et de la terre, au-delà de permanences mythiques, est fréquente dans les fictions narratives du Moyen Âge central. On songe évidemment au Roman d'Énéas, dans lequel Lavine apparaît plus de vingt fois dans le doublet femme/ terre ${ }^{6}$. On pourra également penser au jeu de mot de la Prise d'Orange où Orable/ Orange devient après son baptême et son mariage Guibourg/Guillaume-bourg. Guillaume a pris possession de la ville (le bourg) en épousant la femme, étroitement

5 Art. cit., p. 293.

${ }^{6}$ M. Rousse, «Le pouvoir, la prouesse et l'amour dans l'Énéas », Relire le Roman d'Énéas, dir. J. Dufournet, Paris, Champion, 1985, pp. 149-167. Voir aussi mon article, «'Relire le Roman d'Énéas'. Roman antique et justice féodale », à paraître dans Mélanges J. Dufournet, dir. L. Pierdominici. 
associée à lui à présent et non plus à Orange. Dans notre roman, Vienne, la fille, est donc totalement identifiée à Vienne la capitale du Dauphiné et elle est la seule héritière. Épouser Vienne, c'est donc aussi prendre possession du domaine et le verbe « posséder » prend ici une ampleur particulière, sexuelle et féodale. Ces remarques pourraient paraître quelque peu gratuites et tenant du jeu de mot et d'esprit, mais le déroulement de ce roman, que je qualifierai volontiers de 'matrimonial', démontre que tout n'est pas hasard dans ce choix onomastique.

À peine le cadre du récit a-t-il été posé que le narrateur parle de mariage ${ }^{7}$ : la belle Vienne a douze ans et sa réputation a déjà fait le tour du royaume et au-delà, si bien que « demandee estoit par fame de moult grant prince, barons et chevaliers de France » (p. 58). Jouant de l'entrelacement, l'auteur de Paris et Vienne va mettre en parallèle de la vie de la jeune Vienne les aventures de Paris, fils d'un vassal du dauphin, " ung moult noble chevalier de haut lignage », un certain Jacques, particulièrement aimé du dauphin. Ce Jacques avait un fils, nommé Paris, qui reçut une excellente éducation ; celle-ci est présentée d'une façon fort différente des romans de chevalerie ; si, en effet, il est bien dit que le jeune homme apprend l'art des armes, la première mention de son éducation concerne les « bonnes coustumes » et les lettres (p. 58). Nous sommes au $\mathrm{XV}^{\mathrm{e}}$ siècle et voici donc un fils de l'aristocratie qui fréquente l'école. Le terme " coustumes » ici employé peut être interprété dans ce sens ; certes, il peut simplement renvoyer à une bonne éducation, le garçon ayant la connaissance des usages et bonnes mœurs. Mais, à la fin du Moyen Âge, il est de plus en plus fréquent que les fils de la noblesse suivent des études de droit. Par ailleurs, à la date où ce roman est composé, les coutumes ont fait l'objet de multiples rédactions, depuis la fin du XIII e siècle; en outre, 1432 n'est guère éloignée de la date où, sur ordre de Charles VII, elles vont connaître leur première rédaction officielle, suite à l'ordonnance de Montils-les-Tours, en 1454, qui sanctionne un intérêt particulier et un souci d'organisation de ces règles. On peut donc raisonnablement penser que Pierre de $\mathrm{La}$ Cépède a voulu tracer la silhouette d'un jeune bachelier parfaitement éduqué, ayant suivi des études de lettres - ce qui signifie qu'il connaît le latin - et étant quelque peu frotté de droit. Paris a un ami très cher, nommé Édouard, qui va partager toutes ses aventures. Tous deux sont d'un âge identique, et ils font leur bachelerie ensemble, acquérant ainsi grande réputation, ce que le narrateur expédie très rapidement, en une formule quelque peu lapidaire : « aloient ensemble aulx joustes et tournoyemens qui se faisoient par le monde ou ces deulx chevaliers conqueroient toujours grant fame » (p. 59). Il est en outre précisé que tous deux avaient un brin de voix et que Paris chantait mieux qu'Édouard. Le lecteur apprend dans la foulée que, en dépit de son ignorance de l'amour, Paris savait depuis un an qu'il était amoureux de Vienne. À ce stade $\mathrm{du}$ récit, soit encore au premier paragraphe de l'édition Babbi, on voit comment tout est condensé, comment les éléments essentiels sont rapidement posés. En particulier, le lecteur ignore tout de la naissance de l'amour de Paris, mais il apparaît clairement

\footnotetext{
${ }^{7}$ Dès le premier $\S$ du texte, selon le découpage de éd. cit.
} 
que cet amour sera au centre du roman. Or, ce qui impose le premier suspens dans la question amoureuse n'est pas, selon différents topoï, l'interrogation sur le sentiment amoureux, une aventure de rupture ou un concurrent déloyal, mais un problème tout à fait moderne, qui éloigne de la fin'amor la problématique de Paris et Vienne. Loin de vouloir conquérir une dame de haut rang, Paris prend conscience de la différence sociale existant entre Vienne et lui, et décide que ses sentiments doivent rester secrets pour tous, à l'exception de son cher ami Édouard : « Mais Paris se penssoit tousjours en son cuer que celle amour n'estoit que commune amour, car il n'estoit pas de si grant lignee comme Vienne » (p. 59). Le roman va donc reposer sur une problématique socio-juridique : comment épouser la fille de son suzerain, sachant a priori qu'il s'agit d'une mésalliance?

Quant à Vienne, elle aussi va découvrir l'amour, bien qu'elle n'eût au départ pas la moindre conscience des passions que sa beauté pouvait susciter : " Mais Vienne de tout cecy ne c'epercevoit que Paris fust amoureulx d'elle, ne qu'elle faisoit des aultres chevaliers et barons qui pour l'amour d'elle faisoient tousjours festes et chevaleries ». Si les autres chevaliers restent dans la tradition, en voulant, comme le sous-entend le narrateur, démontrer leur prouesse, Paris choisit une autre façon de faire ; paradoxalement, alors qu'il avait déclaré vouloir que cet amour restât secret, il décide de donner aubade à la belle Vienne. Mais s'agit-il vraiment d'un paradoxe ? Je pencherais plus volontiers vers une articulation consciente de la part de l'auteur qui pose une question d'union matrimoniale au travers du prisme d'une fiction à caractère chevaleresque, mais complètement décalée par rapport aux motifs traditionnels du genre romanesque. Le roman se déroule ainsi selon deux plans : celui de l'action chevaleresque d'une part et, d'autre part, selon un arrière-fond reposant sur la question de l'amour et de la mésalliance. C'est la juxtaposition de ces deux plans et la crise que cette juxtaposition implique qui sera le ressort du roman, dont la fin devra apporter la résolution des tensions et conflits.

Vienne écoutant ces ménestrels qui, à la nuit tomber, viennent charmer ses oreilles - et, par là même, celles de ses parents - en conclut, selon ce qu'elle révèle à sa compagne Ysabel, qu' ' il ne venent si non pour l'amour de moy » (p. 60). Sa curiosité est aussitôt éveillée et elle demande à son père de tout mettre en œuvre pour savoir qui vient ainsi chanter et jouer avec autant de talent. Dix hommes sont alors mis aux aguets afin de conduire, de gré ou de force, les musiciens devant le dauphin. La méthode douce se heurtant au refus de Paris, on met la main à l'épée et la valeur des deux compagnons est alors manifeste, puisqu'ils mettent en déroute les dix hommes qui, en piteux état, vont faire leur rapport au dauphin le lendemain matin. Suite à cette aventure, Vienne comprend aussitôt que «c'estoit quelque jeune homme de grant vertuz qui estoit amoureulx d'elle» (p. 62); sous-entendu, cette vertu touche donc à la fois à l'art de plaire par la musique et à celui de s'imposer par les armes. Paris décide, après l'embuscade ratée, de disparaître et il va se mettre au service de l'évêque de Saint-Laurent. Cette fuite est significative du statut 'moderne' de Paris qui ne part 
pas le moins du monde dans une quelconque quête chevaleresque : le héros est somme toute mi-clerc, mi-chevalier et, effectivement, c'est le clerc qui se rend auprès de l'évêque qui « luy enseignoit choses sainctes et devotes » et avec qui il « passoit temps a grant pensement $»$ (p. 62).

Vienne de son côté est très triste de ne pas savoir qui sont ces ménestrels ; la curiosité déçue cache évidemment un amour naissant qui va aussi tramer le déroulement du récit. Le dauphin, entièrement tourné vers les désirs et humeurs de sa fille unique, décide alors d'organiser des joutes pour la distraire. On revient ici à un motif traditionnel. Les chevaliers joutent devant Vienne et Ysabel, la prouesse devant s'étaler aux yeux de la belle et promise jeune fille. Vienne en est parfaitement consciente et avoue rapidement à sa compagne - qui a remarqué l'amour et la valeur d'Édouard fils du roi d'Angleterre - que deux chevaliers anonymes lui paraissent les plus conformes à ses pensées : « ses deulx chevaliers blans qui ne portent nulles armes a leur escusse approchent mieulx a ma volonté que nulz aultres » (p. 64). On aura reconnu le motif bien connu, depuis le Chevalier de la charrette, du chevalier anonyme participant incognito au tournoi. Le secret doit continuer et nous avons ici un détournement de ce motif de la fin'amor, Paris, plus que des médisants, se gardant tout simplement des conséquences d'un amour socialement impossible. Le chevalier blanc, alias Paris, emporte le prix du tournoi, augmentant le courroux du dauphin qui se retrouve une nouvelle fois devant un 'échec d'identité'. Mais la logique souterraine de la narration suit son cours et Vienne a une nouvelle révélation, en unissant, comme son cœur le lui suggère, les deux pertes d'identité en une seule inconnue :

Par ma foy, belle seur, celuy qui enporta l'escu de crestail et mon chappeau et eust l'onneur des joustes est celuy qui tant m'ayme et chantoit tant doucement devant nostre chambre, car le cuer le me dist (p. 67).

Peut alors se mettre en place un chassé-croisé amoureux dans lequel Vienne et Paris souffrent pareillement du mal d'amour sans qu'aucune issue ne puisse être trouvée, Vienne ignorant tout de l'identité de celui qui a ému son cœur et Paris désirant garder le secret de ses sentiments. Le père de Paris va cependant s'alarmer à la suite de ces joutes, qui revêtent un rôle important d'embrayeur : en effet monseigneur Jacques est fort étonné de l'absence de son fils à ces joutes de son suzerain et, constatant qu'il passe le plus clair de son temps en compagnie de l'évêque, il soupçonne Paris de vouloir entrer en religion, ce qui le désespère. Si l'opposition clergie/chevalerie est totalement effacée par le personnage de Paris, le juvenis, en revanche elle réapparaît chez le senior, qui serait ici le porteur de valeurs plus traditionnelles - bien qu'il ait tenu à l'excellente et interdisciplinaire éducation de son fils. Notons au passage que cette crainte peut être mise en rapport avec la problématique centrale du texte ; si Paris devient homme de religion, il ne saurait se marier et le seigneur Jacques n'aurait pas d'espoir en un futur héritier de son lignage. C'est ce qu'il avoue, alors qu'il est tombé malade du souci que lui donne Paris, à la femme du dauphin, Diane, venu le réconforter sur l'ordre de son mari : 
Et, quant il furent en la chambre ou missere Jacques estoit mallade, madame Dyana luy demanda de son mal, et il respond que toult son mal estoit pour ce que n'avoit veu joyeulx son filz depuis long temps, mais : - [Il] va tousjours avec se dyable d'evesque de Saint Laurens et me fait grant paour, madame, qu'il ne [se] fasse homme de religion, et je n'ay enffant de luy... (p. 79).

Cette visite à monseigneur Jacques malade va être l'occasion d'apporter à Vienne la preuve de ses intuitions. La mère de Paris voulant honorer les dames, elle leur fait en effet les honneurs du château, fort beau et richement meublé ; Vienne découvre alors la chambre de Paris et sous un prétexte de faiblesse momentanée, demande à s'y reposer un instant avec Isabelle. Les deux demoiselles découvrent alors l'oratoire de Paris, dans lequel sont accrochés les trophées qu'il a remportés au tournoi. Elle s'épanche alors encore davantage sur Isabelle et la raisonnable suivante lui rappelle la réalité : elle doit taire ses sentiments et être car ce jeune chevalier, en dépit de sa valeur, n'est pas pour elle :

Douce dame, je vous prie moult doucement que vous ne deissiés chose qui vous puisse tourner a folye et vueillés user de vostre bon sens, car, nonobstant que Paris ait tant de bien, toutesfoys devez considerer qu'il n'est pas egal en vous en lignaige ni en estat, car bien sçavez que moult nobles et grans princes vous ont demandé pour femme et vous ayment que font grant chose pour vous ; et auxi car il est vostre vassal et subgiet et ne fait mestier a vous (p. 81).

Isabelle se fait donc ici l'avocate des principes de l'aristocratie qui se cristallisent fortement à la fin du Moyen Âge pour devenir une constante de l'Ancien Régime. Vienne quant à elle représente une héroïne destinée à une longue postérité littéraire, mettant au premier rang le sentiment amoureux, y compris en contradiction avec les conventions sociales. Elle défend donc ardemment sa position devant Isabelle, en déclarant que rien ne la fera changer d'avis et que la valeur de Paris efface toute barrière sociale. Son amour est irrévocable, dût-elle en perdre la vie. On rejoint également ici un topos à l'œuvre chez bien des auteurs de la fin du Moyen Âge, tels Eustache Deschamps ou Christine de Pizan, comme quoi la noblesse de cœur est la vraie noblesse.

Vienne, tout à son amour, emporte sous sa robe le trophée de Paris et ce dernier, lorsqu'il revient de sa campagne de prouesses en Brabant, découvre la perte et, demandant à sa mère si quelqu'un a pu visiter sa chambre, il prend soupçon que ce pût être la fille du dauphin. Cette dernière, toujours plus alourdie du fardeau de son amour et de son larcin, décide d'aller se confesser à l'évêque de Saint-Laurent. C'est l'occasion, en fait, de demander au prélat de revenir au palais en compagnie de Paris car certaines affaires lui ont été dérobées et la personne coupable, prise de remord, a demandé à Vienne de les remettre à son légitime propriétaire. Paris va donc enfin se retrouver en présence de Vienne qui va l'interroger de façon serrée et obtenir des aveux complet de son amant, depuis les aubades jusqu'aux exploits chevaleresques. Les aveux de Paris sont sans cesse ponctués de formules d'humilité se rapportant à son rang inférieur, «nonobstant que je suis homme de simple estat », « « indigne serviteur et vassal vostre », etc. Mais Vienne semble bien n'accorder aucune importance à la chose et elle finit par lui avouer un amour que, dit-elle, elle confirmera par écrit 
le lendemain. On pourrait considérer que la mention de l'écrit prend ici valeur d'un engagement légal, de véritable fiançailles ${ }^{8}$, mais à caractère totalement clandestin, sans le moindre accord parental.

Le temps passe ainsi et, Vienne ayant atteint l'âge de quinze ans, son père décide de la marier. Paris, apprenant la nouvelle, se plaint à sa chère Vienne et lui rappelle sa promesse. Celle-ci lui répond alors de manière très significative, en termes de droit canonique : son père a toute légitimité pour vouloir la marier mais, déclare-t-elle « Toutefoys, se il ne m'a pas fait consentir au mariage - et vous sçavez bien que mariage n'est nul sans consentement de deulx parties » (p. 88). Vienne est donc bien au courant du droit canonique en affaire de mariage et elle énonce la règle qui va être au cœur de ce roman, autour de laquelle l'action va se construire. Plus précisément la suite des événements dépend très étroitement du conflit qui existe entre pratique de 'aristocratie et droit canonique', le dauphin voulant à tout prix obtenir le consentement de sa fille pour une union que lui a choisie.

Rappelons que, à l'époque où écrit Pierre de La Cépède, les affaires matrimoniales sont entièrement du ressort de l'Église, comme le rappelle déjà à plusieurs reprises, à la fin du XIII siècle, Philippe de Beaumanoir dans ses Coutumes de Beauvaisis :

Li secons cas du quel la juridicions apartient a sainte Église, c'est le mariage (I, 315, p. 154).

Tuit sachent que li mariages qui est tenus pour bons par tesmoignage de sainte Église ne puet estre debatus ne corrompus en court laie (I, 586, p. 285).

Et aussi comme nous avons dit que li tesmoignages de sainte Église doit estre creus de ce qu'ele tesmoigne les bons mariages ou les mauvés... (I, 588, p. 287) .

Gratien précise bien, dans son Decretum : Ubi non est consensus utriusque, non est coniugium (p. II, c. 30, q. 2) ; et le théologien Pierre Lombard affirme de même dans son Liber sententiarum : Efficiens autem causa matrimonii est consensus ${ }^{10}$.

Revenons à notre roman ; Paris, désespéré, avoue son amour à son père et lui demande d'intervenir auprès du dauphin afin qu'il lui accorde la main de sa fille : la patria potestas est donc respectée, comme d'ailleurs dans le cas de Vienne qui avait

${ }^{8}$ Pierre Lombard, par exemple, distingue deux étapes dans le mariage, héritier en cela non seulement de Gratien, mais avant tout des théologiens français du début du siècle, Anselme de Laon, Guillaume de Champeaux et surtout Hugues de Saint-Victor, qui a développé sa doctrine du mariage en particulier dans le De beatae Mariae virginitate et dans le De sacramentis. La première étape du mariage est constituée par les sponsalia : les futurs sont dits alors sponsi, c'est-à-dire une promesse de mariage qui engage juridiquement les futurs pour l'avenir.

${ }^{9}$ Philippe de Beaumanoir, Coutumes de Beauvaisis, éd. A. Salmon, t. 1, Paris, Picard, 1899. On pourra être surpris de trouver ici et plus loin des références à des textes juridiques du XIII ${ }^{e}$ siècle. Mais rappelons que l'époque postérieure, jusqu'au Concile de Trente, ajoutera peu en matière de droit matrimonial, surtout après Latran IV (1215); le principe consensuel est complètement adopté et la copula carnalis, que l'on trouvait chez Hincmar à l'époque carolingienne ou qui était partie intégrante de l'école bolonaise (on le voit aux hésitations de Gratien), n'est plus une condition de l'union matrimoniale reconnue par l'Église.

${ }^{10}$ PL 192, c. 910. 
bien dit qu'elle se soumettait à l'autorité de son père, mais pouvait, dans le cadre de celle-ci, jouer sur les règles du droit canonique. Messire Jacques, apparemment très conscient des conventions sociales, croit devenir fou à entendre son fils mais, à force de supplication de ce dernier, il finit par céder et va faire sa requête auprès du dauphin. L'entrevue entre le suzerain et son vassal estimée se fait sur le mode de la requête, terme employé par le narrateur (p. 90). Celle-ci est faite selon toutes règles et convenances d'humilité. Mais le dauphin, apprenant la nature de cette requête, entre en une rage terrible et, «meu de grant felonnie », il chasse durement monseigneur Jacques : «Villain vassal que vous estes, ainsy me gardez vous mon honneur! Par Dieu, je vous chastiroy de dire telles follies ». On remarquera ici l'emploi du terme « honneur » qui signifie à la fois l'honneur au sens moderne, mais aussi le fief, les possessions. Donner sa fille unique est pour le dauphin surtout une question d'héritage et de politique et cette colère souligne bien le partage entre le sentiment et le pacte social, pivot du mariage aristocratique. D'ailleurs, lorsqu'il s'agit effectivement de trouver un mari à Vienne, son père s'adresse d'abord au comte de Flandres pour lui demander conseil ; ce dernier propose le choix entre le fils du roi d'Angleterre et le fils du duc de Bourgogne, et conseille au dauphin de consulter le roi de France. « De quoy le roi eust grant plaisir et luy va mander que le mariage luy plairoit mieulx avec son nepveu le filz du duc de Bourgogne» (p. 103).

Face à la terrible fin de non-recevoir essuyé par son père - qui d'ailleurs sera plus tard déchu de son fief et jeté en prison-, Paris, sur les conseils de monseigneur Jacques, décide de quitter le Dauphiné, non sans avoir auparavant pris congé de celle qu'il aime. C'est alors que Vienne, en véritable héroïne d'épopée, prend les choses en main et propose à Paris de l'enlever. Au mariage impossible est substitué un rapt. Mais Vienne met une condition, qui rappelle la légalité, en demandant « honnesteté de ma personne joucques a nostre mariage « (p. 92). Vienne tient à son honneur, mais elle tient aussi à une union légale et elle sait fort bien que le droit canonique n'accepte plus la copula carnalis comme validation de mariage ${ }^{11}$, comme le précisent, entre autres, les Décrétales de Grégoire IX : Nisus ad copulam non facit sponsalia de futuro transire in matrimonium (1. 4, tit. 1, chap. 32), pas plus que le rapt puisse être un argument légal. Le récit est donc une nouvelle fois anté sur une question de droit, cette fois-ci liée au raptus. On sait que ce dernier faisait partie de la coutume germanique $^{12}$ et que l'Église eut bien du mal à lutter contre ${ }^{13}$. À la fin du XIII ${ }^{\mathrm{e}}$ siècle,

11 Voir à ce sujet, J. Gaudemet, Le Mariage en Occident. Les mours et le droit, Paris, Cerf, 1987 ; Ph. Toxé, « La copula carnalis chez les canonistes médiévaux », Mariage et sexualité au Moyen Âge : accord ou crise?, dir. M. Rouche, Paris, PUPS, 2000, pp. 123-34.

12 J.-P. Poly, Le Chemin des amours barbares. Genèse médiévale de la sexualité européenne, Paris, Perrin, 2003 ; E. Demougeot, « Le conubium dans les lois barbares », RDE, 12, 1983, pp. 69-82.

13 Sur le rapt à la fin du Moyen Âge, voir H. Benveniste, « Les enlèvements : stratégies matrimoniales, discours juridique et discours politique en France à la fin du Moyen Âge », Revue historique, 283, 1990, pp. 13-35. Voir également, sur les contradictions par rapport au rapt, B. Ribémont, Sexe et amour au Moyen Âge, Paris, Klincksieck, 2009, p. 43. 
Le raptus fait partie de la liste des empêchements dirimants au mariage établie par Hostiensis.

Mais cette tentative de rapt échoue devant les moyens mis en place par le dauphin pour retrouver sa fille : la raison l'emporte et les amants décident, une fois leurs serments de fidélité échangés, de se séparer : Vienne reviendra auprès de son père et tentera d'obtenir le pardon et Paris fuira en d'autres terres avec espoir que le temps permettra aux tensions de se résoudre. Il apparaît clairement avec cet épisode que, si l'auteur entend jouer avec différentes situations romanesques liées à l'amour entre deux jeunes êtres, il manifeste une évidente volonté de rester dans une légalité de droit.

Le récit passe alors véritablement au roman d'aventures, teinté de roman épistolaire, avec ses ingrédients : voyage en mer, combats, Paris faisant ses preuves en terre infidèle, etc. La narration entrelacée se déroule sur deux plans géographiques, au centre desquels il y a Paris d'un côté, Vienne de l'autre. Dans l'univers de Paris, nous sommes dans le roman d'aventure. Celui de Vienne est largement tramé par la question du mariage et du droit, car le dauphin va essayer tous les moyens, y compris les plus cruels, pour obtenir le consentement de sa fille. Il est clair que, pour La Cépède, un mariage imposé exclusivement par l'autorité parentale est impossible. Vienne va alors imaginer un stratagème faisant croire à une terrible maladie qui repoussera tous les prétendants (elle se laisse pourrir des morceaux de volaille sous les aisselles) ${ }^{14}$; le dauphin partira en terre lointaine, sera fait prisonnier à Alexandrie et sera délivré grâce à un Paris devenu maure qui revient auprès de Vienne ; après quelque malentendu à caractère théâtral, il se fait reconnaître. Le dauphin lui accorde son pardon et lui donne la main de sa fille. Tout finira dans la plus grande joie des noces, puis avec l'évocation dernière d'un avenir radieux où Vienne aura trois enfants - deux fils et une fille - et Paris deviendra dauphin de Viennois. Auparavant, cette fois-ci sans le moindre trouble, le dauphin Godefroy « colloca moult noblement a mariage » la fille de Paris. Les coutumes de l'aristocratie et les règles du droit matrimonial de l'Église se rejoignent donc in fine dans une durée dépassant le cadre strict des aventures et dans un espace du compromis.

Ce roman, souvent très moderne comme on peut le mesurer par sa forme partielle épistolaire, joue donc, bien plus que sur les aventures, sur une question de droit matrimonial. Comme tout récit romanesque, il se noue sur des schémas de tension et de rupture appelant résolution. L'auteur a choisi clairement d'écrire ce que je nommerais volontiers un 'roman matrimonial' reposant sur des plans qui interagissent de façon contradictoire, puis conjuguée lorsque les conflits se résolvent, en mêlant et en retravaillant des motifs relevant de la topique du roman de chevalerie/roman d'aventures et une réflexion s'articulant autour du droit matrimonial. Ce texte porte témoignage de la façon dont des questions de droit peuvent travailler en profondeur un texte fictionnel et comment, à la fin du Moyen Âge, un auteur, sans doute au fait d'un mini-

${ }^{14}$ Voir J.-J. Vincensini, art. cit. 
mum de droit, peut jouer avec celui-ci. En termes de realia, Paris et Vienne est un texte révélateur de la complexité des questions d'union matrimoniale prises entre le droit canonique - et donc le consentement mutuel qui s'impose peu à peu à partir du XII ${ }^{\mathrm{e}}$ siècle - et les préoccupations féodales de l'aristocratie. Ce sera un des sujets de tension forte pour les siècles d'Ancien Régime à venir qui donnera naissance, entre autres, à la théorie du 'rapt de séduction'.

\section{BIBLIOGRAPHIE}

Beaumanoir de, O. (1899). Coutumes de Beauvaisis, t. 1, Amédée Salmon (éd.). Paris : Picard.

Cépède de la, P. (1992). Paris et Vienne (version courte), A.M. Babbi (éd.). Milano : Francoangeli. Jean, G. (1987). Le Mariage en Occident. Les mours et le droit. Paris : Cerf.

Lot-Borodine, M. (1913 (1972)). Le Roman idyllique au Moyen Âge. Paris : Picard (Genève : Slatkine).

Ribémont, B. (2009). Sexe et amour au Moyen Âge. Paris : Klincksieck.

Vincensini, J.-J. (1999). Désordre de l'abjection et ordre de la courtoisie: le corps abject dans Paris et Vienne de La Cépède. Medium Aevum, 68, 292-304. 